\title{
Hans-Peter Schwintowski
}

\section{Neue europäische Rechtsprechung zum Vergaberecht}

\author{
Europäisches Vergaberecht; In-House-Geschäft; Rechtsschutz; Schadensersatz; \\ TREA/Halle; Zuschlagskriterien
}

Seit 1999 - der europarechtskonformen Umsetzung des europäischen Vergaberechts in deutsches Recht ${ }^{l}$ - haben zahlreiche Entscheidungen des Europäischen Gerichtshofs Einfluss auf dieses Rechtgebiet genommen und entscheidende Weichen gestellt. Besonders hervorzuheben sind hierbei die erste Entscheidung zum Thema Inhouse-Geschäfte vom November 1999 - die Teckal-Entscheidung ${ }^{2}$-, die erste Entscheidung zu der Frage der vergaberechtlichen Behandlung von Dienstleistungskonzessionen - die TelaustriaEntscheidung ${ }^{3}$ - oder zahlreiche Entscheidungen zum Begriff des öffentlichen Auftraggebers. ${ }^{4}$ Mit diesem Beitrag soll ein Überblick über die neuere Rechtsprechung des EuGH seit 2004 gegeben werden. ${ }^{5}$

\section{Anwendungsbereich - Ausnahmen}

Der Europäische Gerichtshof hat am 11. Januar 2005 ein bemerkenswertes Urteil zu den Grenzen des Anwendungsbereichs des Vergaberechts, genauer zu der Frage, wann ein Inhouse-Geschäft vorliegt, gefällt. ${ }^{6}$ Die Entscheidung könnte klarer kaum ausfallen: ist neben dem öffentlichen Auftraggeber ein privates Unternehmen an einem potenziellen Auftragnehmer gesellschaftsrechtlich beteiligt, so scheidet ein Inhouse-Geschäft aus der Auftrag muss öffentlich ausgeschrieben werden. ${ }^{7}$ Im Ergebnis steht durch die Entscheidung fest, dass alle klassischen öffentlichen Auftraggeber im Sinne des $\S 98$ Nr. 1 GWB - also vor allen Dingen Städte und Gemeinden - bspw. beim Strombezug für gemeindliche Liegenschaften ihren Energiebedarf europaweit ausschreiben müssen, wenn an ihrem Stadtwerk, das die Stadt beliefern soll, ein privates Unternehmen (z.B. RWE/ EnBW/ Vattenfall oder E.ON) beteilig ist. Nur Stadtwerke, die sich zu $100 \%$ im Besitz einer (oder mehrerer) Städte oder Gemeinden befinden, sind auch in Zukunft von der Ausschreibungspflicht befreit, insoweit liegt ein echtes Inhouse-Geschäft vor.

1 Die sog. haushaltsrechtliche Lösung wurde wegen erheblicher Umsetzungsmängel insbesondere hinsichtlich des effektiven Rechtsschutzes vor Zuschlagserteilung zum 1. Januar 1999 durch das Vergaberechtsänderungsgesetz abgelöst.

2 EuGH, Urteil vom 18. November 1999 - Rs. C-107/98

3 „Telaustria und Telefonadress“ EuGH, Urteil vom 7. Dezember 2000 - Rs. C-324/98; vgl dazu die Darstellung der praktischen Umsetzung bei Ortlieb (2001), S. $379 \mathrm{ff}$.

4 Z.B. bezogen auf Messegesellschaften Rs. C-223/99 und C-260/99

5 Ich danke Frau RAin Birgit Ortlieb für konstruktive Mitarbeit und Diskussion.

6 EuGH v. 11. Januar 2005, WuW 2005, 237, EuZW 2005, 86.

7 Dies deckt sich mit der von Schwintowski vertretenen Auffassung, dass „,beteiligte Private an den (Monopol-) Renditen, die beim Marktversagen naturgemäß entstehen, keinesfalls partizipieren dürfen. Vgl. Schwintowski (2004), S. 371. 
In der Entscheidung ging es um das Vergabenachprüfungsverfahren der Arbeitsgemeinschaft Thermische Restabfall- und Energieverwertungsanlage TREA Leuna gegen die Stadt Halle. Die Stadt Halle hatte der RPL Recycling Park Lochau GmbH - ihrer Enkelgesellschaft - den Auftrag im Bereich der Abfallentsorgung ohne öffentliche Ausschreibung vergeben. Dagegen wehrte sich TREA im Vergabenachprüfungsverfahren. TREA machte geltend, dass die Stadt Halle den an RPL vergebenen Auftrag hätte öffentlich ausschreiben müssen, da die Stadt Halle über ihre $100 \%$ ige Tochtergesellschaft nur zu $75,1 \%$ an ihrer Enkelgesellschaft RPL beteiligt sei, während die restlichen 24,9 \% von einer privaten $\mathrm{GmbH}$ gehalten werden.

Die Vergabekammer (VK) gab dem Antrag von TREA statt, weil die Entscheidungen des öffentlichen Auftraggebers auch dann der Nachprüfung zugänglich sein müssten, wenn kein Vergabeverfahren durchgeführt worden sei. Sie stellte ferner fest, dass im vorliegenden Fall keine Rede von einem Inhouse-Geschäft sein könne, weil die private Minderheitsbeteiligung die Schwelle von $10 \%$ überschreite, und nach den deutschen Vorschriften über die $\mathrm{GmbH}$ eine Minderheit vorliege, die bestimmte Rechte habe. Auf Beschwerde der Stadt Halle hat das OLG Naumburg das Verfahren ausgesetzt und dem EuGH eine Reihe von Fragen zur Vorabentscheidung (Art. 234 EG) vorgelegt, die der EuGH wie nachfolgend dargestellt, entschieden hat.

\section{Die Entscheidung, kein Vergabeverfahren einzuleiten, ist nachprüfbar}

Mit der ersten Reihe von Fragen will das OLG Naumburg wissen, ob sich das europäische Vergaberecht auch auf Entscheidungen außerhalb eines förmlichen Vergabeverfahrens, insbesondere auf solche im Vorfeld einer förmlichen Ausschreibung, erstreckt. Von welchem Zeitpunkt an muss ein Mitgliedstaat einem Bieter, Bewerber oder Interessenten den Zugang zu einem Nachprüfungsverfahren eröffnen? Auszulegen sind Art. 1 Abs. 1 der Rechtsmittelrichtlinie RL 89/ 665 und der persönliche oder sachliche Anwendungsbereich der Dienstleistungskoordinierungsrichtlinie RL 92/ 50.

Der Gerichtshof weist darauf hin, dass er bereits früher entschieden habe, dass die dem Vertragsabschluss vorausgehende Entscheidung des öffentlichen Auftraggebers darüber, welchem Bieter der Auftrag erteilt werde, unabhängig von der Möglichkeit, nach dem Vertragsabschluss Schadensersatz zu erlangen, in jedem Fall nachprüfbar sein müsse. ${ }^{8}$ Unter Bezugnahme auf das mit der RL 92/ 50 verfolgte Ziel der Beseitigung der Hemmnisse im freien Dienstleistungsverkehr sowie auf die Ziele, den Wortlaut und die Systematik der RL 89/ 665 habe der EuGH ferner bereits entschieden, dass es möglich sein müsse, die Entscheidung des öffentlichen Auftraggebers über den Widerruf der Ausschreibung eines Dienstleistungsauftrags zum Gegenstand eines Nachprüfungsverfahrens zu machen. ${ }^{9}$ Insoweit könne, wie die Generalanwältin ausgeführt habe, ${ }^{10}$ die Entschei-

8 EuGH v. 28. Oktober 1999, Slg 1999 S. I-76, 71, WuW (1999), S. 1262 Alcatel Austria, Rdn. 43.

9 EuGH vom 18. Juni 2002, Slg (2002), S. I-5553, WuW (2002), S. 1137 Hospitalingenieure Krankenhaustechnik, Rdn. 55.

10 Schlussanträge Nr. 23. 
dung des öffentlichen Auftraggebers, kein Vergabeverfahren einzuleiten, als Pendant zu seiner Entscheidung angesehen werden. Beschließe also ein öffentlicher Auftraggeber, kein Vergabeverfahren einzuleiten, weil der Auftrag seiner Auffassung nach nicht in den Anwendungsbereich des Gemeinschaftsrechts fiele, so handele es sich um die erste Entscheidung, die gerichtlich überprüfbar sei. Nicht nachprüfbar seien demgegenüber Handlungen, die eine bloße Vorstudie des Marktes darstellten oder die rein vorbereitend seien und sich im Rahmen der internen Überlegungen des öffentlichen Auftraggebers im Hinblick auf die Vergabe eines öffentlichen Auftrages abspielten. ${ }^{11}$ Alle Handlungen, die über dieses Stadium hinaus gingen und Rechtswirkungen entfalten könnten, seien folglich nachprüfbar - das Vergabeverfahren müsse folglich formal kein bestimmtes Stadium erreicht haben. Nachprüfbar sei insbesondere die Aufnahme konkreter Vertragsverhandlungen mit einem Interessenten. ${ }^{12}$

Erst recht müssten Fälle nachprüfbar sein, in denen eine De-facto-Vergabe stattgefunden habe. Die Auffassung des OLG Düsseldorf, wonach ein Nachprüfungsantrag dann unstatthaft sei, wenn er sich gegen ein bei seiner Einreichung schon beendetes Vergabeverfahren richte, sei jedenfalls mit der Auffassung des Europäischen Gerichtshofs nicht in Einklang zu bringen. ${ }^{13}$ Dies würde nämlich Umgehungsmöglichkeiten Tür und Tor öffnen. Im Übrigen würde eine so enge Auslegung des Begriffs der nachprüfbaren Entscheidung mit Art. 2 Abs. 1 der RL 89/ 665 unvereinbar sein, wonach die Mitgliedstaaten für jede Entscheidung der öffentlichen Auftraggeber ein Verfahren des vorläufigen Rechtsschutzes vorsehen müssten. ${ }^{14}$

\section{Inhouse-Geschäfte}

Der weitere Fragenkomplex, mit dem sich der EuGH zu befassen hatte, betraf die Abgrenzung eines vergabefreien Inhouse-Geschäftes gegenüber der Verpflichtung, öffentliche Aufträge auszuschreiben. Der Gerichtshof verweist zunächst auf seine im Urteil Teckal entwickelten Grundsätze. ${ }^{15}$ In diesem Urteil hatte er entschieden, dass ein Beschaffungsvorgang nicht öffentlich ausgeschrieben werden müsse, wenn der öffentliche Auftraggeber ihn ,im eigenen Hause“, also mit seinen eigenen administrativen, technischen und sonstigen Mitteln erfülle. Ein solches Inhouse-Geschäft liege nach dem Teckal-Urteil auch dann vor, wenn der öffentliche Auftraggeber über den Auftragnehmer eine ähnliche Kontrolle ausübte, wie über seine eigenen Dienststellen und wenn der Auftragnehmer seine Tätigkeit im Wesentlichen für die öffentliche Stelle oder die öffentlichen Stellen verrichte, die seine Anteile innehätten. ${ }^{16}$ In diesem Zusammenhang erinnert der Gerichtshof in der Sache Halle daran, dass es im Fall Teckal um eine $100 \%$-ige Mutter-Tochter-

\footnotetext{
EuGH v. 11. Januar 2005, WuW (2005), 237, 242 Stadt Halle, Rdn. 35.

Kritisch dazu Storr, WuW (2005), 400 (402).

OLG Düsseldorf v. 03. Dezember 2003, WuW (2004), 239 Sperrmüll.

EuGH v. 11. Januar 2005, WuW (2005), 237, 241 Stadt Halle, Rdn. 30.

EuGH v. 18. November 1999, SLG (1999), I-8121 Teckal.

16 EuGH v. 18. November 1999, SLG (1999), I-8121 Teckal, Rdn 50; vgl. auch Ortlieb (2002), S. 29 ff.; Ortlieb (2003), S. 146 ff.
} 
Beziehung ging. Seither war streitig, ob Inhouse-Geschäfte dann und nur dann vorliegen, wenn es um $100 \%$-ige Beteiligungen zwischen öffentlichem Auftraggeber und Auftragnehmer geht oder ob von einem Inhouse-Geschäft auch dann noch gesprochen werden darf, wenn es sich beim Auftragnehmer um ein gemischtwirtschaftliches Unternehmen handelt, an dem ein privates Unternehmen mitbeteiligt ist. Der Europäische Gerichtshof hat diese Frage nun eindeutig verneint. Die - auch nur minderheitliche - Beteiligung eines privaten Unternehmens am Kapital einer Gesellschaft, an der auch der betreffende öffentliche Auftraggeber beteiligt sei, schließe es auf jeden Fall aus, dass der öffentliche Auftraggeber über diese Gesellschaft eine ähnliche Kontrolle ausübe wie über seine eigenen Dienststellen. ${ }^{17}$

Dieses Ergebnis stützt der Gerichtshof auf zwei Argumente. Zum einen würden mit der Anlage von privatem Kapital in einem Unternehmen andere als im öffentlichen Interesse liegende Ziele verfolgt (Rdn. 50). Zum anderen würde die Vergabe eines öffentlichen Auftrags an ein gemischtwirtschaftliches Unternehmen dem beteiligten privaten Unternehmen einen Vorteil gegenüber seinen Konkurrenten verschaffen (Rdn. 51).

Das zweite Argument ist richtig - es ist in der Tat nicht akzeptabel, dass beteiligte Private an den (Monopol-) Renditen, die bei Marktversagen naturgemäß entstehen, partizipieren dürfen. ${ }^{18}$

Überträgt man allerdings diesen Gedanken auf andere öffentliche Auftraggeber, insbesondere auf öffentliche Unternehmen, die als Nachfrager am Markt auftreten, so gilt dieser Gedanke nur, wenn das Unternehmen, das den Auftrag vergibt, wirklich ein öffentliches Unternehmen im Sinne des $\S 98$ Nr. 2 GWB ist. ${ }^{19}$ Das ist dann und nur dann der Fall, wenn dieses Unternehmen im Allgemeininteresse, also auf Märkten tätig ist, die wettbewerblich nicht oder nicht hinreichend funktionieren. ${ }^{20}$ Die entgegengesetzte Auffassung des EuGH wäre mit dem geltenden Primärrecht des Europäischen Vertrages, insbesondere mit Artt. 4, 98 EG nicht vereinbar. Der EuGH verweist in diesem Zusammenhang allerdings selbst darauf, dass in Art. 1 lit. b RL 92/ 50/ EWG lokale Gebietskörperschaften per definitionem öffentliche Auftraggeber sind. ${ }^{21}$ Aus der Rechtsprechung gehe hervor, so der EuGH weiter, dass Art. 1 lit. a dieser Richtlinie nicht zwischen jenen Aufträgen unterscheide, die ein öffentlicher Auftraggeber vergebe, um seine im Allgemeininteresse liegenden Aufgaben zu erfüllen und jenen Aufträgen, die in keinem Zusammenhang mit derartigen Aufgaben stünden. ${ }^{22}$ Diese Interpretation der Richtlinie 92/ 50/ EWG ist mit dem geltenden Primärrecht des Europäischen Vertrags nicht zu vereinbaren. Danach ist der europäische Gesetzgeber dann und nur dann berechtigt, regulierend in das Marktgeschehen einzugreifen, wenn ein Fall der Marktstörung oder des Marktversagens vorliegt. Ein Fall der Marktstörung oder des Marktversagens liegt aber nicht vor, wenn ein öffentliches Unternehmen, z.B. die Stadtwerke München, so wie jedes andere private

17 EuGH v. 11. Januar 2005, WuW (2005), 237, 244 Stadt Halle, Rdn. 49.

18 Schwintowski (2004), S. 371.

19 Vgl. auch die Ausführungen bei Hausmann/Bultmann zu den Auswirkungen der Entscheidung auf Sektorenauftraggeber, NVwZ (2005), S. 377 (379).

20 Vertiefend Schwintowski (2004), S. 371.

21 EuGH v. 18. November 2004 EuZW (2005), 26 Kommission Deutschland.

22 EuGH v. 15. Januar 1998 Slg 1998, I-73 Mannesmann Anlagenbau Austria, Rdn. 32. 
Unternehmen auch am Wettbewerb teilnimmt und darüber hinaus dem Insolvenzrecht voll unterworfen ist. In einem solchen Fall darf ein öffentliches Unternehmen nach Artt. 4, 98 EG nicht dem öffentlichen Auftragsrecht unterworfen werden, weil sonst durch Überregulierung eine Verzerrung des freien und offenen Wettbewerbs im Binnenmarkt verursacht würde. Das öffentliche Unternehmen würde zugleich gegenüber jedem anderen privaten Unternehmen, das unter gleichen Bedingungen am Wettbewerb teilnimmt, diskriminiert sein (Verstoß gegen Art. 12 EG). EG-Richtlinien sind, wie der Gerichtshof selbst in ständiger Rechtsprechung ausspricht, ihrerseits am Primärrecht zu messen und entsprechend zu korrigieren. ${ }^{23}$ Das gilt selbstverständlich auch für die Rechtsprechung des Europäischen Gerichtshofes selbst.

Richtig bleibt dagegen die Einschätzung der Gerichtshofes dann, wenn es um die Frage geht, wann ein Inhouse-Geschäft vorliegt. Insoweit trifft es auch zu, dass ein öffentlicher Auftrag auch dann nicht mehr ,im eigenen Haus“ erfüllt wird, wenn er an eine andere nicht kontrollierte, also selbständige - juristische Einheit vergeben wird. ${ }^{24}$ Das entspricht der in Deutschland herrschenden Auffassung und Praxis. ${ }^{25}$ In der 7. GWB-Novelle wurde die Rechtsprechung des EuGH zu Inhouse-Geschäften (Stadt Halle Entscheidung vom 11. Januar 2005) umgesetzt. Danach können sich öffentliche Auftraggeber untereinander beauftragen, ohne dass es eines Verfahrens bedarf, solange der auftragnehmende öffentliche Auftraggeber im Wesentlichen für den auftraggebenden öffentlichen Auftraggeber oder überhaupt nicht am Markt tätig ist und - das ist besonders wichtig - kein Privater beteiligt ist. Damit dürften etliche Stadtwerkebeteiligungen zu überdenken sein.

\section{Verfahrensart}

Der EuGH hat mit Urteil vom 14. September 2004 noch einmal klar gestellt, dass die Vergabe von öffentlichen Bauaufträgen im Verhandlungsverfahren ohne vorherige Veröffentlichung einer Vergabebekanntmachung nur unter engen Voraussetzungen zulässig ist. Die Beweislast für die außergewöhnlichen Umstände, die die Ausnahme rechtfertigen, obliegt demjenigen, der sich auf sie berufen will. ${ }^{26}$ Die Entscheidung bestätigt zwei Urteile des Gerichtshofes aus den Jahren 1995 und 1996. ${ }^{27}$ Es ging um ergänzende Bauaufträge der Gemeinde Parma zur Fertigstellung von Rückhaltebecken für verschiedene Flüsse im Bereich der italienischen Orte Marano und Cerano. Der Magistrat der Stadt Parma hatte diese Bauaufträge im Verhandlungsverfahren ohne vorherige Veröffentlichung einer Vergabebekanntmachung vergeben und berief sich im von der Kommission angestrengten Vertragsverletzungsverfahren auf Art. 7 Abs. 3 der RL 93/ 37/ EWG. Der

23 EuGHE, C-376/98, 2000, I-8419 Tabakwerbeverbot.

24 EuGH v. 13. Januar 2005, WuW 2005, S. 353 -357, Kommission/Königreich Spanien.

25 OLG Düsseldorf v. 05. Mai 2004, WuW 2004, 864; richtig auch OLG Düsseldorf v. 15. Oktober 2003, WuW 2004, 121; fraglich ist, ob OLG Düsseldorf v. 12. Januar 2004, WuW 2004, 1353 heute noch aufrecht zu erhalten ist.

26 EuGH v. 14. September 2004, WuW 2004, 1349, EuZW 2004, 665 Marano.

27 EuGH v. 18. Mai 1995, SLG 1995, I S. 1249 Kommission/ Italien; EuGH v. 28. März 1996, SLG 1996, I S. 1949 Kommission/ Deutschland. 
Gerichtshof prüfte, ob die Ausnahmen von Art. 7 Abs. 3 b/ c/ e vorliegen und verneinte dies in allen Fällen. Weder technische (III b) noch dringliche, zwingende (III c) noch zeitliche Gründe (III e) vermochten die Vergabe im Verhandlungsverfahren zu rechtfertigen. Art. 7 III e der RL erlaubt die Vergabe von Bauarbeiten im Verhandlungsverfahren „binnen drei Jahren nach Abschluss des ersten Auftrags“. Die italienische Regierung meinte, die Worte "nach Abschluss des ersten Auftrags" bezögen sich auf die Beendigung der Arbeiten, auf die sich der Auftrag bezieht. Der Gerichtshof stellte durch einen Vergleich mit der dänischen, der englischen, der spanischen und der portugiesischen Fassung der Richtlinie klar, dass es in Art. 7 Abs. 3 e der RL nicht um die Beendigung der Arbeiten - die zeitlich völlig ungewiss sein kann; sondern um den Abschluss des jeweils ersten Vertrages geht. Die italienische Regierung hatte sich insoweit auf einen entschuldbaren Irrtum bei der Interpretation von Art. 7 Abs. 3 e der RL berufen. Der Gerichtshof stellte klar, dass sich ein Mitgliedstaat nicht auf einen entschuldbaren Irrtum berufen kann, um einen Verstoß gegen seine Verpflichtungen aus einer Richtlinie zu rechtfertigen. Der Mitgliedstaat kann nämlich stattdessen im Rahmen eines Vertragsverletzungsverfahrens den genauen Umfang der Verpflichtungen der Mitgliedstaaten ermitteln lassen.

\section{Zuschlagskriterien}

\section{Das Kriterium des niedrigsten Preises}

Mit Urteil vom 07. Oktober 2004 hat der EuGH entschieden, dass es im offenen und nichtoffenen Verfahren kein abstraktes und allgemeines Kriterium geben dürfe, wonach der Zuschlag nur auf den niedrigsten Preis erteilt werden darf. ${ }^{28}$ Es ging um die Auslegung von Art. 30 Abs. 1 RL 93/ 37/ EWG Baukoordinierungsrichtlinie. Gegenstand war die Ausschreibung einer Tiefgarage in der italienischen Stadt Brescia. Sie wurde im nichtoffenen Verfahren nach dem Kriterium des wirtschaftlich günstigsten Angebotes durchgeführt. Auf Beschwerde eines Wettbewerbers vertrat die Aufsichtsbehörde die Auffassung, das italienische Gesetz (Nr. 109/ 1994) für öffentliche Bauarbeiten erlaube nur das Kriterium des niedrigsten Preises. Deshalb sei die Ausschreibung vergaberechtswidrig gewesen. Der EuGH entschied im Vorabentscheidungsverfahren (Art. 234 EG), dass ein nationaler Gesetzgeber, der - so wie das italienische Gesetz - ein ausschließliches Kriterium für die Vergabe öffentlicher Bauverträge abstrakt und allgemein festlege, damit Art. 30 Abs. 1 der RL 93/ 37/ EWG verletze. Er nehme nämlich den öffentlichen Auftraggebern die Möglichkeit, die Art und die Besonderheiten derartiger Aufträge im einzelnen zu berücksichtigen, indem sie für jeden von ihnen das Kriterium wählten, das am besten geeignet sei, den freien Wettbewerb zu sichern und so die Auswahl des besten Angebots zu gewährleisten (Urteilsgründe Rdn. 40). 
Der Gerichtshof stellt auch klar, dass es im konkreten Einzelfall sehr wohl zulässig sein könne, die Verwendung des niedrigsten Preises als einziges Zuschlagskriterium vorzuschreiben (Urteilsgründe Rdn. 39). Ganz allgemein gehen die Europäischen Richtlinien aber vom Prinzip des besten Preis-Leistungs-Verhältnisses aus, um die Wirtschaftlichkeit eines Angebotes zu ermitteln. Daher dürfe sich der nationale Gesetzgeber nur nicht allgemein und abstrakt, also losgelöst vom Einzelfall, auf ein einziges Zuschlagskriterium beschränken. Der BGH hat übrigens in diesem Zusammenhang zu Recht darauf hingewiesen, dass die Vergabekriterien vorhersehbar und transparent sein müssen, weil sich die Bieter sonst darauf nicht einstellen könnten. ${ }^{29}$

\section{Vergabefremde Zuschlagskriterien}

Ebenfalls im Rahmen eines Vorabentscheidungsverfahrens erging das viel beachtete Urteil des EuGH vom 04.12.2003 in Sachen Ökostrom. ${ }^{30}$ Es ging um die Belieferung der Verwaltungsdienststellen des Bundes mit elektrischem Strom im österreichischen Bundesland Kärnten. Unter der Rubrik Zuschlagskriterien war angegeben: wirtschaftlich günstigstes Angebot nach den folgenden Kriterien: Umweltgerechtigkeit der Leistungen gem. Ausschreibungsunterlagen.

Der Bieter musste nachweisen, dass er mindestens 22,5 GWh aus erneuerbaren Energien erzeugt oder zugekauft hat. Als Zuschlagskriterien wurden der Nettopreis pro kWh mit $55 \%$ und das Kriterium Energie aus erneuerbaren Energieträgern mit $45 \%$ festgelegt. Es wurde nicht verlangt, dass sich der Bieter zum Nachweis seiner Bezugsquellen zu verpflichten hätte.

Der EuGH hat zunächst auf sein Urteil „Concordia Bus Finland“ hingewiesen und noch einmal betont, dass das wirtschaftlich günstigste Angebot Umweltschutzkriterien berücksichtigen darf, sofern sie mit dem Gegenstand des Auftrags zusammenhängen, dem Auftraggeber keine unbeschränkte Entscheidungsfreiheit einräumen, ausdrücklich im Leistungsverzeichnis oder in der Bekanntmachung des Auftrags genannt sind und bei ihnen alle wesentlichen Grundsätze des Gemeinschaftsrechts, insbesondere das Diskriminierungsverbot, beachtet werden. ${ }^{31}$ Dies steht im Einklang mit der Mitteilung der Europäischen Kommission über die Berücksichtigung von ökologischen Aspekten aus dem Jahr $2003 .^{32}$

Im zweiten Schritt erklärt das Gericht, dass nach ständiger Rechtsprechung die Wahl des wirtschaftlich günstigsten Angebots den öffentlichen Auftraggebern die Entscheidung über die Kriterien für die Zuschlagserteilung überlässt, sofern diese Kriterien der Ermittlung des wirtschaftlich günstigsten Angebots dienen und dem öffentlichen Auftraggeber

29 BGH v. 03. Juni 2004, WuW 2004, 988.

30 EuGH v. 04. März 2003, WuW 2004, 225 Wienstrom, dazu Steinberg (2005), S. 132 ff.; ders. (2004) S. 76; Fischer (2004), S. 492; Odendahl (2004), S. 647.

31 EuGH v. 17. September 2002, SLG 2002, S. I-7213, WuW 2002, 1023 ff. Concordia Bus Finland.

32 Interpretierende Mitteilung der Kommission der Europäischen Gemeinschaft vom 04. Juli 2001 über das auf das öffentliche Auftragswesen anwendbare Gemeinschaftsrecht und die Möglichkeit zur Berücksichtigung von Umweltbelangen bei der Vergabe öffentlicher Aufträge (KOM (2001) 274 endgültig). 
bei der Vergabe des Auftrags an einen Bieter keine uneingeschränkte Entscheidungsfreiheit einräumen. ${ }^{33}$ Die Nutzung erneuerbarer Energiequellen diene dem Umweltschutz, da sie zur Verringerung der Emissionen von Treibhausgasen beitrage, die zu den Hauptursachen der Klimaänderungen zählten, zu deren Bekämpfung sich die Europäische Gemeinschaft und ihre Mitgliedstaaten verpflichtet habe. ${ }^{34}$ Unter diesen Umständen erscheine die $45 \%$-Gewichtung für erneuerbare Energien nicht als Hindernis für eine Gesamtwürdigung der der Ermittlung des wirtschaftlich günstigsten Angebots dienenden Kriterien.

Allerdings - so der EuGH weiter - verlange der Grundsatz der Gleichbehandlung eine objektive und transparente Bewertung der verschiedenen Angebote. Das wiederum setze voraus, dass der öffentliche Auftraggeber in der Lage sei, anhand der von den Bietern gelieferten Angaben und Unterlagen effektiv zu überprüfen, ob ihre Angebote die Zuschlagskriterien erfüllten (Urteilsgründe Rdn. 50). Wenn daher ein öffentlicher Auftraggeber ein Zuschlagskriterium festlege und dabei angebe, dass er weder bereit noch in der Lage sei, die Richtigkeit der Angaben der Bieter zu prüfen, so verstoße er gegen den Grundsatz der Gleichbehandlung, denn ein solches Kriterium gewährleiste nicht die Transparenz und die Objektivität des Vergabeverfahrens (Urteilsgründe Rdn. 51).

Ein Zuschlagskriterium, das sich darüber hinaus auf die Menge Strom aus erneuerbaren Energieträgern beziehe, die den im Rahmen des Auftrags zu erwartenden jährlichen Verbrauch übersteige, könne nicht als mit dem Gegenstand des Auftrags zusammenhängend angesehen werden (Urteilsgründe Rdn. 68). Im Übrigen kann dieses Zuschlagskriterium den Bietern einen Vorteil verschaffen, die wegen ihrer größeren Erzeugungs- oder Lieferkapazitäten in der Lage seien, größere Mengen Strom zu liefern als andere. Dieses Kriterium könne somit zu einer ungerechtfertigten Diskriminierung von Bietern führen, deren Angebot die mit dem Gegenstand des Angebots zusammenhängenden Voraussetzungen ansonsten uneingeschränkt erfülle (Urteilsgründe Rdn. 69).

\section{Rechtsschutz}

\section{Grossmann Air Service}

Im Januar 1998 wurden Bedarfsflüge für die österreichische Bundesregierung und deren Delegationen mit Exklusivjets und Flugzeugen ausgeschrieben. Außer dem Unternehmen Grossmann Air Service bewarb sich niemand - deshalb wurde die erste Ausschreibung im April 1998 widerrufen. Im Juli 1998 wurden die Bedarfsflüge erneut ausgeschrieben. Grossmann Air Service besorgte sich zwar die betreffenden Ausschreibungsdokumente, sah aber von einem Angebot ab. Im Oktober 1998 teilte die österreichische Regierung Grossmann Air Service ihre Absicht mit, den Auftrag an Lauda Air zu vergeben. Grossmann erhielt dieses Schreiben am nächsten Tag (09. Oktober 1998). Der Vertrag mit

33 EuGH v. 20. September 1988, SLG 1988, S. 4635 Beentjes, Rdn. 19, 26; EuGH v. 18. Oktober 2001, SLG 2001, S. I-7725 Siac Construktion Rdn. 36, 37 und Concordia Bus Finnland a.a.O. Rdn. 59, 61.

EuGH v. 13. März 2001, SLG 2001, S. I-2099 PreussenElektra, Rdn. 73. 
Lauda Air wurde am 29. Oktober 1998 geschlossen. Mit Antragsschrift vom 19. Oktober 1998, die am 23. Oktober 1998 zur Post gegeben wurde und am 27. Oktober 1998 beim Bundesvergabeamt einging, stellte Grossmann einen Antrag auf Nachprüfung der Entscheidung, die Flugleistungen an Lauda Air zu vergeben und beantragte die Nichtigerklärung. Im Rahmen des Vorlageverfahrens (Art. 234 EG) war zu klären, ob Grossmann noch antragslegitimiert war, obwohl man nach Benachrichtigung von der Zuschlagsentscheidung (09. Oktober 1998) unter Einreichung eines Nachprüfungsantrags (23. Oktober 1998) einen Zeitraum von 14 Tagen hatte verstreichen lassen, ohne ein Schlichtungsersuchen einzureichen. Der Europäische Gerichtshof entschied, dass eine Person von einem Nachprüfungsverfahren ausgeschlossen werden darf, wenn sie von der ihr eingeräumten Möglichkeit, vor Erteilung des Zuschlags eine Nachprüfung einzuleiten, keinen Gebrauch macht. Dann ist Art. 1 Abs. 2 und Art. 2 Abs. 1 b der Richtlinie 98/ 665/ EWG nicht verletzt. Aus der Tatsache, dass Grossmann es unterlassen hat, vor Einleitung eines Nachprüfungsverfahrens eine Schlichtungsstelle anzurufen, folgt aber nicht, dass das Interesse an einem Auftrag als entfallen gilt. Einer solchen Interpretation würde Art. 1 Abs. 3 der RL 89/ 665 entgegenstehen.

\section{Siemens AG Österreich}

Im Urteil vom 18. März $2004^{35}$ ging es um die Frage, ob das Verbot der Subvergabe ein rechtswidriges Vergabekriterium ist. Der Gerichtshof bejahte dies im Grundsatz, denn Art. 25 der RL 92/ 50 räumt dem Bieter ausdrücklich die Möglichkeit ein, einen Teil des Auftrags an Dritte zu vergeben. Allerdings muss der Bieter nachweisen, dass er tatsächlich über die dem Dritten zustehenden Mittel, die er nicht selbst besitzt und die zur Ausführung des Auftrags erforderlich sind, verfügt. Das entspricht der Auffassung, die der EuGH bereits am 02. Dezember 1999 entschieden hatte. ${ }^{36}$ Hiervon ausgehend muss das innerstaatliche Recht die Möglichkeit bieten, eine mit dem gemeinschaftlichen Vergaberecht unvereinbare Ausschreibungsklausel im Rahmen des durch die Richtlinie 89/ 665 vorgesehenen Nachprüfungsverfahrens zu überprüfen.

35 WuW 2004, 565 Siemens AG Österreich.

36 EuGH v. 02. Dezember 1999, SLG 1999, S. I 8607, WuW 2000, 221 Holst Italia. 


\section{Pacta sunt servanda - Schadensersatz}

Am 09. September 2004 hat der Europäische Gerichtshof ein Urteil gefällt, das für das deutsche Vergaberecht von großer praktischer Bedeutung werden könnte. ${ }^{37}$ Es ging um Müllentsorgungsverträge, die verschiedene Städte im Jahr 1997 geschlossen hatten, ohne das nach der RL 92/ 50 vorgesehene Ausschreibungsverfahren durchzuführen. Die Europäische Kommission rügte dies im Vertragsverletzungsverfahren gegen Deutschland. Die Bundesregierung räumte den Verstoß gegen das Gemeinschaftsrecht ein, wies aber darauf hin, dass sie inzwischen sichergestellt habe, dass künftig gemeinschaftsrechtskonform ausgeschrieben werde. $\mathrm{Zu}$ einer Beendigung der gemeinschaftsrechtswidrig geschlossenen Verträge seien allerdings die deutschen Städte nicht verpflichtet gewesen. Dem widersprach die Kommission. Sie hatte die Bundesrepublik Deutschland am 20. April 2001 in einer mit Gründen versehenen Stellungnahme aufgefordert, die Verträge in einer Frist von zwei Monaten zu beenden. Bei Ablauf dieser Frist seien die bis zum 31. Dezember 2003 geschlossenen Verträge nicht beendet gewesen - der Gemeinschaftsrechtsverstoß habe folglich weiter angedauert.

Der EuGH stimmte der Kommission zu und verwies auf eine vorangegangene Entscheidung vom 10. April 2003. ${ }^{38}$ Die Bundesregierung hatte vorgetragen, dass das Prinzip pacta sunt servanda einer Pflicht zur Beendigung von Liefer- und Bauaufträgen entgegenstehe und auch den Bestand solcher Verträge schütze, die unter Verstoß gegen vergaberechtliche Bestimmungen zustande gekommen seien. Für diese Auffassung stützt sich die Bundesregierung auf Art. 2 Abs. 6 der RL 89/ 665/ EWG. Dem sei - so der EuGH - entgegenzuhalten, dass diese Bestimmung zwar die Mitgliedstaaten ermächtige, nach Vertragsabschluss den nationalen Rechtsschutz auf Schadensersatz für die durch einen solchen Verstoß geschädigten Personen zu begrenzen, dass sie aber nicht dazu führe, dass das Verhalten eines öffentlichen Auftraggebers in jedem Fall im Rahmen einer Vertragsverletzungslage als gemeinschaftsrechtskonform anzusehen sei (Urteilsgründe Rdn. 15). Die Aussage ist klar und dürfte für den Rechtsschutz in der Bundesrepublik Deutschland von einiger Bedeutung sein. Denn die Begrenzung des deutschen Rechtsschutzes auf Schadensersatz bei Vergabeverstößen macht den Vergabeverstoß nicht gemeinschaftsrechtskonform. Das heißt aber, dass der vergaberechtswidrig zustande gekommene Vertrag gegen ein gesetzliches Verbot ( $\$ 134$ BGB) verstößt und folglich nichtig ist. Vergaberechtswidrig zustande gekommene Verträge sind - wenn sie noch nicht erfüllt sind folglich in Zukunft zu beenden und neu auszuschreiben. Die vom OLG Düsseldorf mit Beschluss vom 03. Dezember 2003 entwickelten - und oben schon kritisierten - Grundsätze bei De-facto-Vergaben können demnach nicht mehr aufrecht erhalten werden. Dogmatisch könnte der deutsche Gesetzgeber alles beim Alten belassen, müsste aber klarstellen, dass der Schadensersatz bei Vergaberechtsverstößen prinzipiell auch den Naturalersatz im Sinne des $\S 249$ BGB umfasst, also auf Wiederherstellung des Zustandes

37 EuGH v. 09. September 2004, WuW 2004, 1229 Lüdinghausen.

38 EuGH v. 10. April 2003, SLG 2003, S. I-3609, WuW 2003, 691 Fortdauernder Gemeinschaftsverstoß, Rdn. 35, 36. 
gerichtet ist, der ohne Vergabeverstoß bestand. Das wäre eine Situation, in der der Zuschlag noch nicht erteilt war, in der noch hätte ordnungsgemäß ausgeschrieben werden müssen. Dies entspricht im Übrigen dem Beschluss des Bundesverfassungsgerichts vom 29. Juli 2004, ${ }^{39}$ wonach ein Schaden im Sinne des $§ 107$ Abs. 2 GWB regelmäßig schon darin besteht, dass durch den beanstandeten Vergaberechtsverstoß die Aussichten des antragstellenden Bieters auf den Zuschlag zumindest verschlechtert worden sein könnten. Der mit der 7. Novelle zum GWB geplante neue $\S 101 \mathrm{~b}$ GWB wird erstmals regeln, dass De-facto-Vergaben nicht zu einem wirksamen Vertragsverhältnis führen können, denn Verträge sind nach $\S 101$ b GWB nicht mehr nur von Anfang an unwirksam, wenn die oben erwähnte Informationspflicht unbeachtet geblieben ist, sondern außerdem, wenn „ein öffentlicher Auftrag unmittelbar an ein Unternehmen erteilt (wird), ohne andere Unternehmen am Vergabeverfahren zu beteiligen und ohne dass dies aufgrund dieses Gesetzes (des GWB) gestattet ist".

\section{Literaturverzeichnis}

Fischer, Kristian (2004), Vergabefremde Zwecke im öffentlichen Auftragswesen: Zulässigkeit nach europäischem Gemeinschaftsrecht, in: EuZW, 15. Jg., Heft 16, S. 492-496

Hausmann, Friedrich Ludwig und Peter Friedrich Bultmann (2005), Die Entscheidung des EuGH in der Rechtssache „Stadt Halle”, ein neues Paradigma für vergaberechtsfreie In-House-Geschäfte, in: NVwZ, S. 377-381

Odendahl, Kerstin (2004), Die Berücksichtigung vergabefremder Kriterien im öffentlichen Auftragswesen, in: EuZW, 15. Jg., Heft 21, S. 647-652

Ortlieb, Birgit (2002), Inhouse-Geschäft als Ausnahme zur Ausschreibungspflicht zugleich eine Besprechung des BGH vom 12. Juni 2001, in: Zeitschrift für neues Energierecht, S. 29 ff.

Ortlieb, Birgit (2003), Umfang und Reichweite sog. Inhouse-Geschäfte als Ausnahme zur Ausschreibungspflicht im Spannungsfeld zunehmender Privatisierung - zugleich eine Besprechung der Entscheidung des Bundesgerichtshofes vom 12. Juni 2001, Zeitschrift Wirtschaft und Wettbewerb, S. 146 ff.

Ortlieb Birgit (2001), Vergaberechtliche Beurteilung von Konzessionsverträgen, in: Energiewirtschaft im Aufbruch, Köln, S. 379-392

Schwintowski, Hans-Peter (2004), Konkurrenz der Öffentlichen Hand für privatwirtschaftliche Unternehmen aus der Perspektive des Vergaberechts, ZögU, Band 27, Heft 4, S. 360-376

Steinberg, Philipp (2004), Die „Wienstrom“, Entscheidung des EuGH, in: EuZW 15. Jg., Heft 3, S. $76-78$

Steinberg, Philipp (2005), Vergaberechtliche Steuerung als Verbundaufgabe, Baden-Baden

Storr, Stefan (2005), „De-facto-Vergabe“ und „In-House-Geschäft“ erneut vor dem EuGH, Das Urteil „Stadt Halle“ setzt neue Maßstäbe, in: WuW, Heft 4, S. 400-405

$39 \mathrm{WuW}$ (2004), 1103. 